\title{
Cancer Clinical and Economic Outcomes Shared Resource
}

National Cancer Institute

\section{Source}

National Cancer Institute. Cancer Clinical and Economic Outcomes Shared Resource. NCI

Thesaurus. Code C39342.

The Cancer Clinical and Economic Outcomes Shared Resource is a multi-disciplinary resource providing methodologic expertise and support for Cancer Center investigators in patient-centered evaluation in patients at risk for or with cancer. The main areas of support include: cost and cost-effectiveness analyses of programs and initiatives, quality of life and functional status assessment, psychosocial outcome and satisfaction evaluation, survey design and research, and simulation modeling. 